\title{
METHOTREXATE INDUCED LUNG INJURY
}

Flavio Barboza1, ${ }^{1,}$, Thalyne Aparecida Leite de Lima ${ }^{1}$, Vivian de Aquino Medici ${ }^{1}$, Evelyn Angrevski Rodrigues ${ }^{1}$, Talles Henrique Pichinelli Maffei ${ }^{1}$, Maitê Luise Zanette ${ }^{1}$, Lucas do Carmo de Carvalho ${ }^{1}$, Heloisa Maria Lopes Scarinci ${ }^{1}$, Nohati Rhanda Freitas dos Santos ${ }^{1}$, Bruna Luiza Oliveira Lima ${ }^{1}$, Raquel Gerep Pereira ${ }^{1}$, Eduarda Judith Dias Jacome Silva ${ }^{1}$, Sofia Landim Teixeirense Pinheiro ${ }^{1}$, Ian Jader Alves de Oliveira ${ }^{1}$, Bruna Sayuri Tanaka ${ }^{1}$, Catharine Luísa Rocha Soares ${ }^{1}$

1. Universidade Federal do Mato Grosso, Sinop (MT), Brazil.

*Corresponding author: flavio.fbarboza@uol.com.br

\section{BACKGROUND}

Methotrexate (MTX) is a disease modifying antirheumatic drug (DMARD) widely used for the treatment of rheumatoid arthritis and collagenoses. A variety of adverse events can be associated with MTX, such as nausea, vomiting, headache, urticaria, stomatitis, nodulosis and panniculitis. Other people can experience serious adverse events such as bone marrow aplasia, acute liver failure and MTX-induced pneumonitis, especially in elderly patients. The incidence of MTX- the first year, but late presentation has been published. The main symptoms are cough, shortness of induced pneumonitis varies between 0.3 and $11,6 \%$, occurring mainly during breath and fever. The chest X-ray shows peripheral lung opacities and computed tomography (CT) reveals ground-glass opacities and basal pulmonary fibrosis in advanced cases.

\section{CASE REPORT}

A 72-years old woman with rheumatoid arthritis for 17 years and previously treated with hydroxychloroquine, corticosteroids and nonsteroidal anti-inflammatory presented polyarthritis, rheumatoid nodules and morning stiffness. Laboratory tests: rheumatoid factor 512, anti-CCP antibodies > 200, antinuclear antibodies (ANA) homogeneous pattern 1:160, elevated ESR and CRP. Physical examination: normal breath sound. Normal CT and spirometry. Methotrexate $15 \mathrm{mg}$ and folic acid were given. After the second dose, the patient had progressive shortness of breath. She was diagnosed with pneumonia in the emergency room and was treated with levofloxacin, which didn't result in improvements. The patient scheduled an appointment with rheumatologist and presented coarse crackle lung sounds, HR: 110 and SpO2: 93\%. Computed tomography showed nonspecific interstitial pneumonitis (Fig. 1). After MTX was interrupted and it was increased corticoids, the patient improved her clinical status and CT images were better.

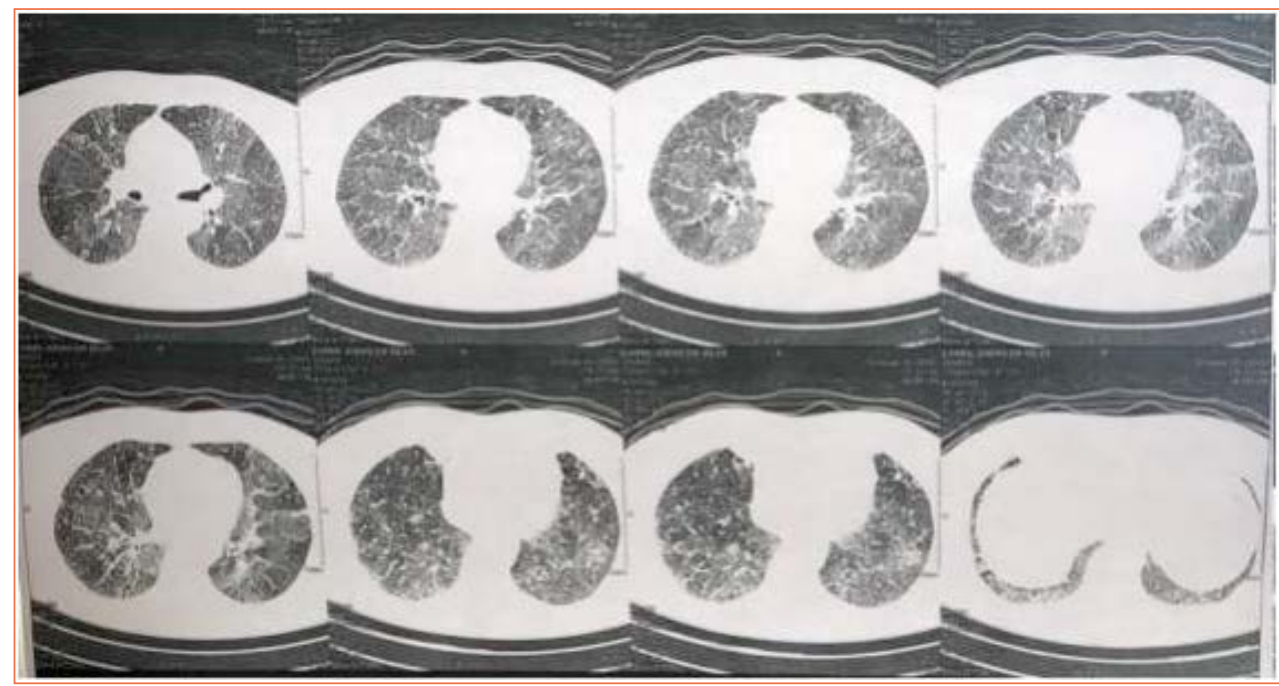

Figure 1. Nonspecific interstitial pneumonitis 


\section{CONCLUSION}

Even though MTX is considered an anchor drug for many diseases, its adverse effects can be life-threatening. It is crucial to follow up patients that are taking MTX, because if they experience shortness breath it could be a symptom of pneumonitis. 